\title{
Caring through science - Visualizing geographic accessibility of health care facilities in areas with high prevalence of comorbidities
}

\author{
Lourens Snyman *a, Serena Coetzee $^{\mathrm{a}}$, Inger Fabris-Rotelli ${ }^{\mathrm{b}}$, Debashis Basu ${ }^{\mathrm{c}}$ \\ ${ }^{a}$ Department of Geography, Geoinformatics and Meteorology, University of Pretoria, Pretoria, South Africa, \{lourens.snyman, \\ serena.coetzee\}@up.ac.za \\ ${ }^{b}$ Department of Statistics, University of Pretoria, Pretoria, South Africa, inger.fabris-rotelli@up.ac.za \\ ${ }^{c}$ Department of Public Health Medicine, University of Pretoria, Pretoria, South Africa, debashis.basu@up.ac.za
}

Keywords: Comorbidities, Accessibility, Mapping, Visualization, Health Care Facilities

\begin{abstract}
:
In the current times, societies are continuously being confronted with the harsh reality of COVID-19. Our attitude towards health systems are influenced by a health facility's ability or non-ability to cope under these new and sometimes unknown challenges. South African health systems comprises of public and private sectors, where the majority of the population $(80 \%)$ are dependent on the public sector. The health system in South Africa, in general, is operating under extreme pressure due to the increasing burden of communicable diseases (such as tuberculosis and HIV) and non-communicable diseases (such as hypertension and diabetes), while adjusting to an ever-increasing demand for health services and trying to be resilient at the same time, i.e. "the capacity of health actors, institutions and populations to prepare for and effectively respond to crises, maintain core functions when a crisis hits; and, informed by lessons learned during this crisis, reorganize if conditions require it" (Kruk, Myers et al. 2015). The South African health system is devolved into district health systems where primary health care clinics and general medical practitioners are the first point of entry. The setting of this study is the Tshwane Health District, one of the 52 districts in South Africa. It has a population of 2.9 million and population density of 480 per square kilometre $\left(\mathrm{km}^{2}\right)$. The unemployment rate is notably high at $24 \%$, with close to $15 \%$ of households have no source of income, strengthening the need to bring health care services closer to people.
\end{abstract}

This study focuses on accessibility of health care facilities in the Tshwane Health District to the population it serves. Knowledge about the location of the most vulnerable and hard-to-reach communities is especially important, and particularly in the context of COVID-19, for the development of effective response and intervention strategies. For the purpose of this study, the occurrence (or prevalence) of COVID-19 comorbidities will be analysed in both space and time in relation to the geographic location of health care facilities. There are 68 primary health care clinics across the district servicing just below 2.5 million people within a 5 kilometre travel distance. From a medical point of view; analysing the prevalence of comorbidities geographically could provide valuable insights regarding priority health care needs and prevailing socio-economic realities of communities. This will allow for a critical evaluation of the current geographic footprint of health care facilities and potentially elicit recommendations for the optimal positioning of facilities, specifically primary health care clinics.

Accessibility refers to "the quality of being easy to approach, reach, enter, speak with, use, or understand" accessibility can therefore be described as how easy it is to approach, reach, enter, consult, use, or understand a particular service. Accessibility is multi-faceted and can be measured in different ways. For example, health care service accessibility has been measured by assessing the costs of health care utilization (affordability), health service compliance and satisfaction (acceptability), adequacy of health service provision (availability), travel impedance between patients and providers (geographic accessibility) and appropriateness and suitability of health services (accommodation) (Neutens 2015). Some researchers distinguish between place-based and people-based measures of accessibility (Miller, 2007; Neutens, 2010; Kwan, 2009). Place-based measures consider the closeness between a key location in an individual's life (e.g. home or workplace) and desired locations (e.g. a service provider location), while people-based measures consider the activities of individuals in space and time, and how they use places in the real and in the virtual world (Miller, 2007). In this abstract, the focus is on geographic accessibility, a form of place-based accessibility, also sometimes referred to as spatial or physical accessibility.

Accessibility based on distance or travel time can be measured from the population or service provider perspective. Population accessibility is indicated by the shortest distance or travel time between the population and a service provider location; a shorter distance or travel time implies better accessibility by the population. Service provider accessibility is indicated by the size of the population within a specified threshold distance or travel time from a service provider location;

\footnotetext{
${ }^{1}$ Dictionary.com
} 
a larger population implies better accessibility of the service provider location. Accordingly, the widely used two-step floating catchment area (2SFCA) method (Luo and Wang, 2003) measures geographic accessibility in terms of both the size of the population catchment (service provider locations that fall within a threshold distance from the population) and the size of the service catchment (population within a threshold distance or travel time from the service provider) (McGrail and Humphreys 2009).

The aim of this study is firstly to assess the availability and geographic accessibility of health care facilities in the Tshwane Health District in areas with a high prevalence of comorbidities. Accessibility is based on both straight-line (Euclidean) and travel network distance calculations. Apart from the accessibility calculations, spatial statistical techniques, such as kernel density estimation, cluster analysis, spatial autocorrelations and location quotients are used to identify and visualize spatial accessibility patterns in both the health care facilities and population data (Shaweno et al. 2018). Secondly, different geospatial visualization techniques are used to display accessibility results. These include hexagon tessellation, choropleth maps, isochrones and Thiessen polygons (or Voronoi diagrams) for visualizing health facility catchment areas and population demand (Kauhl et al. 2016; Widrich et al. 2021; Ye and Kim 2016).

Lastly, the pros and cons of different techniques for communicating accessibility results are compared and evaluated. The overall purpose of the study is to support a more resilient health system by raising awareness of the spatial relationships between health care facilities and potential geographic hot-spots with a high prevalence of comorbidities. Based on this information, expertise and treatment methods could be aligned to better serve these areas. Intervention and response strategies will have a geographic dimension directly linked to both capacity and accessibility of health services.

\section{References}

Kauhl, B., et al. (2016) "Do the risk factors for type 2 diabetes mellitus vary by location? A spatial analysis of health insurance claims in Northeastern Germany using kernel density estimation and geographically weighted regression." Int J Health Geogr 15, 38

Kruk, M. E., et al. (2015). "What is a resilient health system? Lessons from Ebola." The Lancet 385(9980): 1910-1912.

Kwan, M.P., (2009). From place-based to people-based exposure measures. Soc. Sci. Med. 69 (9), 1311-1313.

Luo, W., Wang, F., (2003). "Measures of spatial accessibility to health care in a GIS environment: synthesis and a case study in the Chicago region". Environ. Plann. B: Plann. Des. 30 (6), 865-884.

McGrail, M. R. and J. S. Humphreys (2009). "Measuring spatial accessibility to primary care in rural areas: Improving the effectiveness of the two-step floating catchment area method." Applied Geography 29(4): 533-541.

Miller H. J., (2007). "Place-based versus people-based geographic information science" Geographical Compass 1503 535

Neutens, T., et al. (2010). "Equity of urban service delivery: a comparison of different accessibility measures." Environment \& Planning A 42(7).

Neutens, T. (2015). "Accessibility, equity and health care: review and research directions for transport geographers." Journal of Transport Geography 43: 14-27.

Shaweno, D., et al. (2018) "Methods used in the spatial analysis of tuberculosis epidemiology: a systematic review". BMC Med 16, 193

Widrich, J., et al. (2021). "Geographic Visualization of Mortality in the United States as Related to Healthcare Access by County." Cureus 13(1)

Ye, H. and H. Kim (2016). "Locating healthcare facilities using a network-based covering location problem." GeoJournal 81(6): $875-890$ 